\title{
Measurement of minimum bias collisions, underlying event activity and double-parton scattering
}

\author{
Ankita Mehta* \\ (On behalf of the CMS Collaboration) \\ Panjab University Chandigarh (India) \\ E-mail: ankita.mehta@cern.ch
}

\begin{abstract}
We present recent results on minimum bias collisions, underlying event activity and double-parton scattering using data recorded by the CMS detector at the LHC. The results on the measurement of the underlying event using leading tracks, jets, and Drell-Yan processes are presented. Doubleparton scattering is investigated in several final states including vector bosons and multi-jets, and the results are compared to other experiments and to multi-parton interactions models tuned to recent underlying event measurements at CMS.
\end{abstract}

ICHEP 2018, 39 $9^{\text {th }}$ International Conference on High Energy Physics

4-11 July 2018

Seoul, Republic of Korea

* Speaker, current affiliation : Eötvös Loránd University, Budapest. 


\section{Introduction}

Studies of minimum bias collisions, underlying event (UE) activity and double-parton scattering (DPS) are interesting and challenging. These events are produced by strong interactions of partons inside the hadrons, which occur at low momentum, for which predictions of quantum chromodynamics cannot be obtained perturbatively. Measurements are sensitive to the transition region between hard processes calculable with perturbative techniques and soft processes described by nonperturbative models. Thus, these measurements are required for a full description of particle production in proton-proton ( $\mathrm{pp}$ ) collisions at the LHC. In this note, recent experimental results from such measurements are discussed based on the proton-proton collision data collected using the CMS detector [1] at a center-of-mass energy $\sqrt{\mathrm{s}}=13 \mathrm{TeV}$.

\section{Minimum bias measurements}

The measurement of pseudorapidity $(\eta)$, transverse momentum $\left(\mathrm{p}_{\mathrm{T}}\right)$, and multiplicity distributions for charged particles is performed for three event classes having different topologies of the final state particles [2]. An inelastic (INEL) event sample corresponds to events with activity on one side of the calorimeters. A non-single diffractive enhanced (NSD-enhanced) event sample is defined by requiring activity on both sides of the calorimeter whereas a single diffractive enhanced (SD-enhanced) event sample consists of events with activity on only one side of the calorimeters, with a veto condition being applied to the other side. $\mathrm{p}_{\mathrm{T}}$ distributions of leading charged particle for these event samples are shown in Fig. 1. The predictions of EPOS LHC [5] provide the best description of the data for the INEL and NSD-enhanced event samples within the experimental uncertainties. However, PYTHIA8 CUETM1 [3, 4] gives the best description of the data for SDenhanced event sample whereas EPOS LHC shows a disagreement with the data up to $40 \%$. This measurement not only provides important information on low energy exchange processes dominating pp interactions but can also be used to tune the model parameters in existing event generators.
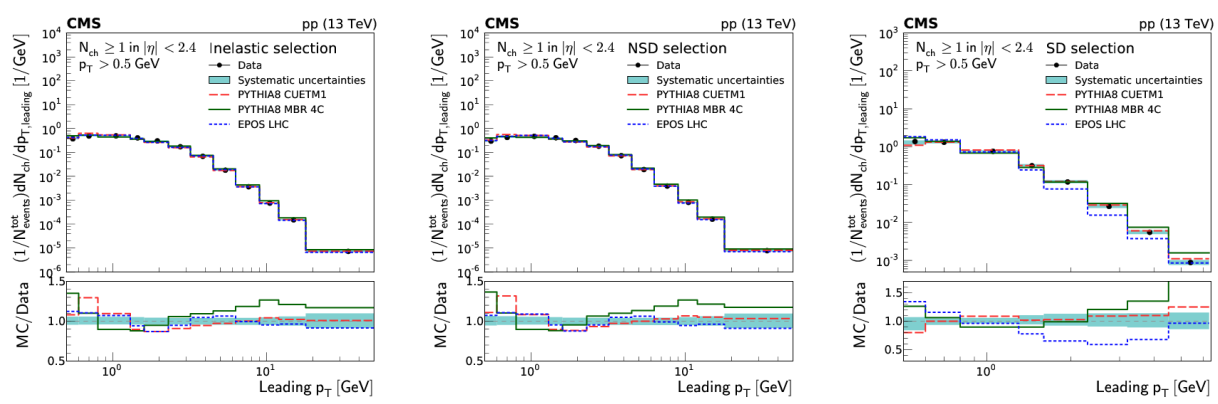

Figure 1: Charged particle transverse momentum densities for inelastic, NSD-enhanced, and SD-enhanced event samples [2].

A femtoscopic analysis of Bose-Einstein correlations has been performed to probe the size and shape of the particle emitting region for different charged particle multiplicities [6]. Correlation functions are used to find the 1-D lengths of homogeneity $\left(\mathrm{R}_{\text {inv }}\right)$. Results are studied as functions of the event multiplicity $\left(\mathrm{N}_{\mathrm{trk}}^{\text {offline }}\right)$, average pair transverse momentum $\left(\mathrm{k}_{\mathrm{T}}\right)$ and pair transverse mass 
$\left(\mathrm{m}_{\mathrm{T}}\right)$. Figure 2 (left) shows $\mathrm{R}_{\mathrm{inv}}$ as a function of $\mathrm{k}_{\mathrm{T}}$ in two multiplicity bins, minimum bias (MB) with $\mathrm{N}_{\text {trk }}^{\text {offline }}<80$ and high multiplicity $(\mathrm{HM})$ with $\mathrm{N}_{\text {trk }}^{\text {offine }}>80 . \mathrm{R}_{\text {inv }}$ tends to decrease with increasing $\mathrm{k}_{\mathrm{T}}$, more rapidly at lower multiplicities. This behavior is compatible with an emitting source that was expanding prior to decoupling. Valuable information about the collective transverse expansion of the system can be obtained from the slope of a linear fit to $1 / R_{\text {inv }}^{2}$ versus $m_{T}$. Figure 2 (right) shows $1 / \mathrm{R}_{\mathrm{inv}}^{2}$ versus $\mathrm{m}_{\mathrm{T}}$ for a variety of multiplicity ranges. The collective flow decreases with increasing multiplicity, but this trend seems to saturate around a reconstructed track multiplicity of 80 . These observations are consistent with those obtained using high-multiplicity events in relativistic AA collisions.
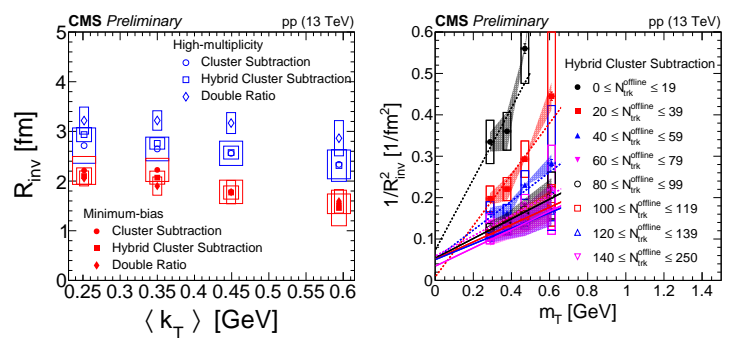

Figure 2: $R_{i n v}$ from the three methods as a function of $k_{T}(l e f t) .1 / R_{i n v}^{2}$ as a function of $m_{T}$ for the hybrid cluster subtraction (HCS) method (right). Statistical uncertainties are represented by error bars, systematic uncertainties related to the HCS method are shown as open boxes and the relative uncertainties from the intramethods variation are represented by the shaded bands. Only statistical uncertainties are considered in all the fits [6].

\section{Underlying event activity}

The measurements of UE activity based on events with a $\mathrm{Z}$ boson, decaying into a pair of muons, as leading object are presented. The UE activity is quantified using observables such as charged particle multiplicity $\left(\mathrm{N}_{\mathrm{ch}}\right)$ and scalar sum of $\mathrm{p}_{\mathrm{T}}$ of the charged particles $\left(\sum \mathrm{p}_{\mathrm{T}}\right)$, as a function of $\mathrm{p}_{\mathrm{T}}$ of the dimuon system $\left(\mathrm{p}_{\mathrm{T}}^{\mu \mu}\right)$. Different phase space regions are defined based on the azimuthal angular separation $(\Delta \phi)$ between a charged particle and the $\mathrm{Z}$ boson direction. The transverse $\left(60^{\circ}<|\Delta \phi|<120^{\circ}\right)$ and towards $\left(|\Delta \phi|<60^{\circ}\right)$ regions are sensitive to the multi-parton interactions (MPI), radiative contributions and modeling of the UE activity, while the recoil activity dominates in the away region $\left(|\Delta \phi|>120^{\circ}\right)$ [7]. The collision energy evolution of the UE activity in Z boson events is quantified in Figure 3 (left) shows the ratio of UE activity at different collision energies for the data and the simulations. An increase of $25 \%$ in the particle density is observed as the collision energy increases from 7 to $13 \mathrm{TeV}$ [8]. This behavior is quantitatively well described by POWHEG+PYTHIA8 [9] and POWHEG+HERWIG++ [10] event generators. To further quantify the energy dependence of the UE activity, events with $\mathrm{p}_{\mathrm{T}}^{\mu \mu}<5 \mathrm{GeV}$ are studied and shown in Fig. 3

(right). Setting an upper limit on $\mathrm{p}_{\mathrm{T}}^{\mu \mu}$ reduces the initial state and final state radiation contributions and the remaining UE activity stems mainly from MPI [8][11]. There is a significant increase as the collision energy rises from 1.96 to $13 \mathrm{TeV}$, which is better described by POWHEG with PYTHIA8. The comparison of the distributions with and without MPI indicates that the radiation contributions, which increase slowly with $\sqrt{\mathrm{s}}$, are small. 

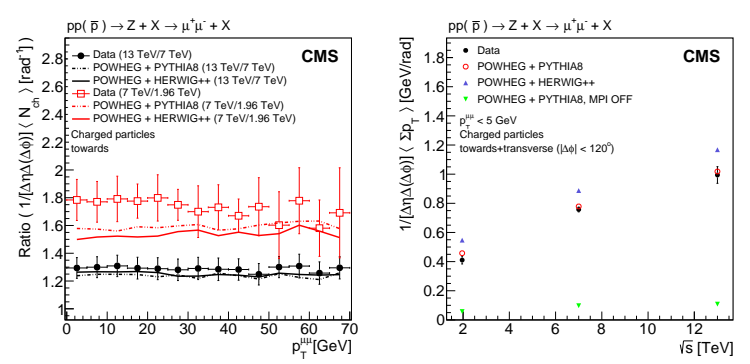

Figure 3: Comparison of UE activity in $\mathrm{Z}$ boson events at different center-of-mass energies for $\mathrm{N}_{\mathrm{ch}}$ density in the towards region (left). Comparison of $\sum \mathrm{p}_{\mathrm{T}}$ density for $\mathrm{Z}$ boson events with $\mathrm{p}_{\mathrm{T}}^{\mu \mu}<5 \mathrm{GeV}$ as a function of $\sqrt{\mathrm{s}}$ for data, and predictions from simulations (right). The error bars represent the statistical and systematic uncertainties added in quadrature [7].

\section{Double-parton scattering}

The DPS cross section is measured using the same-sign $\mathrm{W}$ boson pair production with two $\mathrm{W}$ bosons decaying leptonically into a pair of muons or an electron-muon pair [12]. A multivariate analysis based on boosted decision trees (BDT) is used to discriminate the signal and background processes. BDTs are trained using a set of lepton kinematic variables, defined based on the topological differences between the DPS signal and background processes. To maximize the sensitivity of the signal process, the statistical analysis is performed by categorizing the shape of BDT discriminant into different charge configurations of the final state leptons. The obtained results are also found to be consistent with predictions from PYTHIA8 and those obtained using the factorization approach [13] with a $\sigma_{\text {eff }}$ of $20.7 \pm 6.6 \mathrm{mb}$ as measured in $\mathrm{W}+2$ jets final state at $7 \mathrm{TeV}$ [14].

\section{References}

[1] CMS Collaboration, JINST 3 S08004 (2008).

[2] CMS Collaboration, Eur. Phys. J. C 78697 (2018).

[3] T. Sjöstrand et.al., Comput. Phys. Commun. 178, 852 (2008).

[4] CMS Collaboration, Eur. Phys. J. C 76, 155 (2016).

[5] T. Pierog, Phys. Rev. C 92, 034906 (2015).

[6] CMS Collaboration, CMS-PAS-FSQ-15-009 (2018).

[7] CMS Collaboration, JHEP 07032 (2018).

[8] CMS Collaboration, Eur. Phys. J. C 722080 (2012).

[9] S. Frixione et.al., JHEP JHEP 11070 (2001).

[10] M. Bähr et.al., Eur. Phys. J. C 58639 (2008).

[11] R. Kumar et.al., Phys. Rev. D 935 (2016).

[12] CMS Collaboration, CMS-PAS-FSQ-16-009 (2017).

[13] J.R. Gaunt et al., Eur. Phys. J. C 6953 (2010).

[14] CMS Collaboration, JHEP 1403032 (2014). 